\title{
Percepción de empoderamiento comunitario en las mujeres madre cabeza de familia de la ciudad de Cúcuta durante el primer semestre de 2007
}

\author{
Luz Marina Bautista Rodrígueł* Maritza Lisset Martínez Carvajal** y Angélica Mallely Gómez Castilla***
}

Recibido:

22 de febrero de 2008

Aceptado:

20 de junio de 2008

\section{Resumen}

Se describe la percepción de empoderamiento comunitario en las mujeres madre cabeza de familia de la ciudad de Cúcuta durante el primer semestre de 2007, el estudio es cuantitativo, de tipo descriptivo, retrospectivo de corte transversal que se llevó a cabo con la utilización de una escala de empoderamiento, instrumento creado por Gladys Eugenia Canaval, Enf., M.Sc., Ph.D., Profesora Titular, Escuela de Enfermería, Facultad de Salud, Universidad del Valle, Cali; el cual cuenta con 27 enunciados que se basan en una escala tipo Likert de cinco puntos que van desde fuertemente de acuerdo (calificación de 5) hasta fuertemente en desacuerdo (calificación de 1), este instrumento busca medir la percepción del empoderamiento comunitario evaluando cuatro factores: sentido de comunidad, involucramiento, participación e influencia. La muestra la conformaron 315 mujeres madre cabeza de familia pertenecientes a las 10 comunas de la zona urbana de la ciudad de Cúcuta quienes a partir del 26 de Junio del 2007 iniciaron el diligenciamiento del instrumento. Como resultado se pudo determinar que la organización comunitaria y formación de coaliciones son una condición influyente en el empoderamiento de las mujeres reflejado en que las mujeres del estudio muestran una alta percepción de empoderamiento comunitario donde de cada 100 mujeres 63 presentaron puntajes por encima del $70 \%$.

Palabras Claves: Percepción, empoderamiento comunitario, mujeres madres cabeza de familia

\section{Abstract}

The primary target of the study was to describe the Perception of communitarian empowerment in the household's women-mother-head in the Cúcuta's city during the first semester of the 2007, being a descriptive study, cross-sectional
*Enfermera. Magíster en Enfermería con énfasis en Gerencia de Servicios de

Salud Universidad Nacional UFPS. Miembro Grupo Investigación Cuidado. E-mail: bautrod@gmail.com.

** Estudiante de VIII Semestre de Enfermería, Universidad Francisco de Paula Santander. E-mail: marylyss@hotmail.com

*** Estudiante de VIII Semestre de Enfermería, Universidad Francisco de Paula Santander.

E-mail: diosmesonrie@hotmail.com 
and retrospective type of quantitative cut that was carried out with the use of a empowerment's scale, instrument created by Gladys Eugenia Canaval, Enf., M.Sc., Ph.D., Professor Titular, School of Infirmary, Faculty of Health, University of the Valle, Cali; which counts with 27 statements that are based on a scale Likert type of five points that go from strongly in agreement (qualification of 5) to strongly in discord (qualification of 1) and that it looks for to measure the perception of communitarian empowerment evaluating four factors: sense of community, involvement, participation and influences. The sample was conform by 315 household's women-mother-head from the 10 communes of the urban zone of the Cúcuta's city who the 26 of June of the 2007 began to fill up the instruments. As result could be determined that the communitarian organization and formation of coalitions are an influential condition in the empowerment of the women reflected in which the women of the study show a high perception of communitarian empowerment where of each 100 women 63 presented scores over $70 \%$.

Keyswords: Perception, community empowerment, women heads of households mothers

\section{Introducción}

$\mathrm{H}$

oy se reconoce que las mujeres tienen un papel importante en la construcción de una sociedad igual, justa y respetuosa como también lo tienen en la promoción de la salud y la prevención de la enfermedad ${ }^{1}$, pero esta labor se ve opacada por el patriarcalismo y las inequidades de género existentes en la sociedad que limitan el potencial de empoderamiento dentro de los procesos relacionados con la salud de la mujer y sus familias ${ }^{2}$, y aunque los avances logrados en los últimos años han producido una transformación en las condiciones de vida de una alta proporción de mujeres el impacto de estos cambios en su participación en las estructuras de toma de decisiones ha sido débil ${ }^{3}$

Desafortunadamente en nuestro país no se cuenta con la valoración cuantitativa del número de mujeres que han asumido este empoderamiento, nilapercepciónadquiridadel mismo, siendo la ausencia de datos estadísticos que determinen el empoderamiento en las mujeres colombianas un obstáculo que hace aún más difícil tener conocimiento real de la situación de las mismas, interfiriendo de igual manera en el planteamiento de estrategias y medidas que contribuyan al incremento del empoderamiento en este grupo poblacional.

\footnotetext{
${ }^{1}$ SALINAS, Paulina. Ciudadanía y género: mujeres y liderazgo en las provincias de Antofagasta, el Loa y Tocopilla [en línea]. 2005 Junio [citado en 03 jun. 2006]. Disponible en internet: URL:http//www2.udec.cl/serví/numero5/ion04.htm.

${ }^{2}$ CEPAL. Políticas para el empoderamiento de las mujeres como estrategia de la lucha contra la pobreza [en línea]. 2001 Octubre [citado en 16 feb. 2006]. Disponible de Internet: URL:http://www.cepal.org/politicas/mujer

${ }^{3}$ CANAVAL, Gladys Eugenia. Promoción de la salud: Predictores de empoderamiento comunitario en mujeres [en línea]. 1996 Noviembre [citado en 30 nov. 2005]. Disponible en internet: URL:http://Tone.Udea.edu.co/revista/html/modules.php?op=modl oad\&name $=$ sechons \&file $=$ index \& req $=$ viewartide\&ardid $=171>$ Pág. 15
} 
Entre los pocos estudios que se han realizado acerca del tema se encuentran los predictores de empoderamiento comunitario en mujeres, realizado en Cali en cuyos resultados se encontró que las mujeres no cuentan con los recursos necesarios para moldear su participación, cuentan con empleos de muy bajo nivel que no les permiten desarrollar habilidades que contribuyan al incremento de su participación en actividades empoderadoras, el $65 \%$ de las mujeres no reportó actividades empoderadoras; el $45 \%$ no tuvo ningún modelo de acción comunitaria; y el 97\% no participó en grupos organizados. ${ }^{4}$

A pesar de esta dificultad actualmente se reconoce la importancia de fortalecer el empoderamiento comunitario en las mujeres, pero se realizan muy pocas acciones para lograrlo, dentro de estas se destacan las actividades realizadas con las madres cabeza de familia uno de los grupos vulnerables quienes a pesar de su situación de marginalización y discriminación han logrado desarrollar habilidades empoderadoras a través de su organización en asociaciones adscritas a entes gubernamentales que han permitido mejorar su desempeño dentro de la comunidad, incrementando su grado de participación y liderazgo en las diferentes actividades y decisiones vitales para su reconocimiento por parte de la sociedad. ${ }^{5}$

Por esta razón es de vital importancia el desarrollo de este estudio cuyo propósito es el de brindar datos reales que contribuyan en la toma de medidas dirigidas a conseguir el empoderamiento de las mujeres en dos sentidos: de reconocimiento de sus propias capacidades para ejercer influencia, poder y liderazgo, así como de actuar en función de ese reconocimiento, es decir, de ejercer efectivamente esos tres ejes primordiales

\section{Objetivo general}

Describir la percepción de empoderamiento comunitario en las mujeres madres cabeza de familia adscritas a las asociaciones regidas por la secretaría de la equidad y participación de la mujer en la ciudad de Cúcuta durante el primer semestre de 2007.

\section{Objetivo específico}

Identificar el sentido de comunidad, involucramiento, participación comunitaria e influencia existente en las mujeres madres cabeza de familia adscritas a las asociaciones regidas por la secretaría de la equidad y participación de la mujer, en la ciudad de Cúcuta durante el primer semestre de 2007.

\section{Materiales y métodos}

La muestra se obtuvo de una población de 14165 mujeres madre cabeza de familia adscritas a la Secretaría de la Equidad y Participación de la ciudad de Cúcuta, conformada por 315 mujeres pertenecientes a las 10 comunas de la zona urbana de la misma en los sectores de Barrio Popular, Antonia Santos, Carlos Pizarro, Gaitán Parte Alta, La Florida, Policarpa, Belén, El Contento, Alto del Pamplonita y Sevilla.

\footnotetext{
${ }^{4}$ Convención sobre la eliminación de todas las formas de discriminación contra la mujer. Congreso de Colombia. Ley 51 de 1981. Reglamentada por el Decreto nacional 1398 de 1990. Asamblea General de las Naciones Unidas el 18 de diciembre de 1979 y firmada en Copenhague el 17 de julio de 1980".

${ }^{5}$ CONGRESO DE COLOMBIA. LEY 82 de 1993. normas para apoyar de manera especial a la mujer cabeza de familia.
} 
Para la muestra se define la mujer madre cabeza de familia "quien siendo soltera o casada, tenga bajo su cargo, económico o socialmente, en forma permanente, hijos menores propios $\mathrm{u}$ otras personas incapaces $\mathrm{o}$ incapacitadas para trabajar, ya sea por ausencia permanente o incapacidad física, sensorial, síquica o moral del cónyuge o compañero permanente o deficiencia sustancial de ayuda de los demás miembros del núcleo familiar" ${ }^{\prime \prime}$

Teniendo en cuenta que el tamaño de la población era muy grande se trató como una población infinita, utilizándose la fórmula de muestreo indicada para este caso con un nivel de confiabilidad del $96 \%$ y un error de 0.05 determinándose así el tamaño de la muestra.

El proceso de tabulación y análisis se llevó a cabo a través de medidas de dispersión, distribución de frecuencias y porcentajes representados en tablas y gráficos clasificados de acuerdo a los objetivos planteados en el estudio.

Este estudio por incluir vidas humanas se ha fundamentado filosóficamente sobre los principios éticos, resaltándose que es una investigación de bajo riesgo basado en los principios éticos de respeto a la dignidad humana, beneficencia, no-maleficencia y justicia. Las mujeres pertenecientes a la muestra aprobaron su participación a través de un consentimiento informado escrito donde se plantean los objetivos del estudio, las condiciones bajo las cuales se realizó el mismo y los beneficios que conlleva su desarrollo.
Se utilizó el instrumento denominado Escala percepción de empoderamiento, creado por Gladys Eugenia Canaval, Enf., M.Sc., Ph.D., Profesora Titular, Escuela de Enfermería, Facultad de Salud, Universidad del Valle, Cali; el instrumento cuenta con una parte inicial que contempla variables socio demográficas como: edad, ocupación, estado civil, escolaridad, número de hijos, procedencia, estrato, seguridad social, barrio y comuna. La segunda sección cuenta con 27 enunciados los cuales buscan medir la percepción del empoderamiento comunitario evaluando cuatro factores: sentido de comunidad (2 ítems), involucramiento (7 ítems), participación (7 ítems) e influencia $(11 \text { ítems })^{7}$

En el empoderamiento comunitario es importante definir variables en las que se desglosa su concepto las cuales son sentido de comunidad, involucramiento, participación e influencia.

Sentido de comunidad. Si es importante para las mujeres y más aun si comparten objetivos e intereses con las personas que conforman su comunidad. El sentido sicológico de comunidad como lo plantea Sarason citado Jariego Isidro M (2005) "es una experiencia subjetiva de pertenencia a una colectividad mayor, formando parte de una red de relaciones de apoyo mutuo en la que se puede confiar" ${ }^{8}$

Los elementos que le dan forma a esta valoración personal son la percepción de similitud con otros, el reconocimiento de la

${ }^{6}$ COLOMBIA. Consejería Presidencial para la Equidad de la Mujer. Ley 823 del 2003 [en línea]. 2003 Febrero [citado en 03 feb. 2007]. Disponible en internet: URL:http://www. presidencia.gov.co/equidad/acuerdo.htm

${ }^{7}$ CANAVAL, Gladys Eugenia. Promoción de la salud: Predictores de empoderamiento comunitario en mujeres [en línea]. 1996 Noviembre [citado en 30 nov. 2005]. Disponible en internet: URL:http://Tone.Udea.edu.co/revista/html/modules.php?op=mod load\&name $=$ sechons \&file $=$ index $\&$ req $=$ viewartide $\&$ ardid $=171$

8JARIEGO, Isidro Maya. Sentido de comunidad y potenciación comunitaria [en línea]. 2005 Julio Pág. 189 [citado en 16 jul. 2007]. Disponible en Internet: URL: http://66.102.1.104/scholar?hl=es\&lr=\&q=cache:-dMkYEnWPAJ:dialnet.unirioja.es/servlet/citart\%3Finfo\%3Dlink\%26codigo\%3D1197378\%26orden\%3D55238+ 
interdependencia con los demás, la voluntad de mantener esa interdependencia dando o haciendo por otros lo que uno espera de ellos, y el sentimiento de que uno es parte de una estructura más amplia, estable y fiable. "Es así como el sentido de comunidad tiene un núcleo importante en torno a la interacción social entre los miembros de un colectivo, y se complementa con la percepción de arraigo territorial y un sentimiento general de mutualidad e interdependencia (Sánchez Vidal, citado por Jariego Isidro 2005)” ${ }^{\prime}$.

Involucramiento. Ser parte activa de, hacer parte activa de, formar parte activa de, también tiene que ver, en primer lugar, con los valores compartidos por los miembros del grupo y en segundo lugar con el intercambio de recursos para satisfacer las necesidades de los integrantes.

Consiste en el sentimiento de haber invertido parte de sí mismo en la comunidad, y de pertenecer a ella. Concretamente, esto conlleva la delimitación de una frontera entre los miembros y los que no lo son, la existencia de un sistema de símbolos compartidos, la experiencia de seguridad emocional, la inversión personal en la comunidad y, finalmente, el sentimiento de pertenencia, identificación e involucramiento.

Participación. Laparticipaciónsignificaque todos los actores sociales de una comunidad toman parte en las deliberaciones y decisiones sobre cualquier problema que afecta a la comunidad incluyendo las decisiones sobre necesidades y prioridades, la asunción de las responsabilidades y obligaciones para la formulación de planes y adopción de medidas y evaluación de los resultados. Ser parte de los grupos tomadores de decisión, tomar parte en las decisiones y en los cambios, identificar necesidades comunes, identificar los recursos y actuar a favor del colectivo.

A nivel municipal, la participación comunitaria es la organización racional y consciente de las personas que habitan en un municipio, con el propósito de proponer las iniciativas que satisfagan sus necesidades; definir interesesyvalorescomunes; colaboraren la realización de obras y prestación de servicios públicos; conocer sus responsabilidades como miembros del municipio e influir en la toma de decisiones del grupo.

Influencia. Se hace relevante la influencia reciproca entre los miembros y la comunidad donde se experimentan dinámicas de intercambio reciproco de poder, que se analizan a través de los resultados obtenidos y de la participación individual con otras personas para conseguir determinados objetivos y ejercer control. $\mathrm{O}$ bien, mejorando la estructura para facilitar la participación de sus miembros y aumentar su efectividad. Por último toma parte importante la acción colectiva para mejorar la calidad de vida.

Hace referencia al poder que los miembros ejercen sobre el colectivo, y recíprocamente al poder de las dinámicas del grupo sobre sus miembros. Las personas se sienten más atraídas por los grupos en los que pueden ejercer influencia. En esta línea, no es infrecuente encontrar en las comunidades una estructura centro-periferia, en la que los individuos con un sentimiento más fuerte de comunidad son las más activos en las dinámicas del grupo y se convierten en un referente para el resto de participantes. ${ }^{10}$

Las respuestas a los 27 enunciados se basan en una escala tipo Likert de cinco 
puntos que van desde fuertemente de acuerdo (calificación de 5) hasta fuertemente en desacuerdo (calificación de 1). Un número impar de opción de respuesta implica un punto central neutral. La escala de cinco puntos permite una adecuada discriminación entre categorías y variabilidad de las respuestas. La suma del puntaje total de la escala tiene un rango de 27 a 135 puntos. Los puntaje altos indican gran empoderamiento comunitario, esta variable se manejara en base al promedio teniéndose en cuenta que si los puntajes totales se encuentran por encima del $70 \%$ se dice que es alto, si las cifras se encuentran entre el 40 y el $69 \%$ se dice que es medio y si son iguales a $39 \%$ o por debajo de este se dice que es bajo.

En el estudio de Propiedades psicométricas de una escala para medir percepción del empoderamiento comunitario en mujeres se demostró que la escala de empoderamiento es un instrumento válido y confiable que puede ser usado en investigaciones futuras (La Confiabilidad de la escala con el uso del coeficiente alfa de Cronbach indicó que produce datos consistentes internamente. La consistencia interna de la escala con 27 enunciados fue alta $(\alpha=0.95)$ ). La escala de empoderamiento puede llegar a ser un instrumentoútilen rastreoo como instrumento diagnóstico y también para evaluar el efecto de intervenciones específicas. ${ }^{11}$

\section{Resultados y discusión}

\section{Percepción de las mujeres madre cabeza de familia frente al sentido de comunidad}

Las mujeres encuestadas demuestran que se sienten parte de una colectividad. Esto se evidencia en el cuadro 1, donde se observa que el $77 \%$ de las mujeres opinaron estar de acuerdo y fuertemente de acuerdo en que "las mujeres de su barrio tienen intereses comunes" y un $80 \%$ expresa estar en acuerdo y fuertemente en acuerdo en que "es importante compartir esos intereses". Se reconoce en la muestra la percepción de similitud con otros, el reconocimiento de la interdependencia con los demás y la voluntad de mantener esa interdependencia que genere efectos beneficiosos para todas y es así como la sensación de formar parte de un grupo se convierte en un sentimiento compartido de que las necesidades colectivas deben ser afrontadas bajo un trabajo cooperativo entre todos sus integrantes.

Cuadro 1. Distribución de la percepción de las mujeres madres cabeza de familia frente al sentido de comunidad. Cúcuta 2007.

\begin{tabular}{|l|c|c|c|c|c|c|c|c|c|c|}
\hline \multirow{2}{*}{ ITEMS } & \multicolumn{2}{|c|}{ FD } & \multicolumn{2}{c|}{ D } & \multicolumn{2}{c|}{ I } & \multicolumn{2}{c|}{ DA } & \multicolumn{2}{c|}{ FDA } \\
\cline { 2 - 10 } & $\mathrm{F}$ & $\%$ & $\mathrm{~F}$ & $\%$ & $\mathrm{~F}$ & $\%$ & $\mathrm{~F}$ & $\%$ & $\mathrm{~F}$ & $\%$ \\
\hline $\begin{array}{l}\text { Intereses comunes } \\
\text { con otras personas }\end{array}$ & 18 & $6 \%$ & 21 & $7 \%$ & 33 & $10 \%$ & 119 & $38 \%$ & 124 & $39 \%$ \\
\hline $\begin{array}{l}\text { Importancia de } \\
\text { compartir intereses } \\
\text { comunes }\end{array}$ & 6 & $2 \%$ & 29 & $9 \%$ & 28 & $9 \%$ & 125 & $40 \%$ & 127 & $40 \%$ \\
\hline
\end{tabular}

FD: Fuertemente en desacuerdo D: En desacuerdo I: Indecisa DA: De acuerdo FDA: Fuertemente de acuerdo F: Frecuencia \%: Porcentaje.

Cabe resaltar que el principal interés que comparten las encuestadas, es el haber tenido que asumir por diferentes motivos el cuidado y mantenimiento de sus hogares, colocándolas en situaciones similares que ponen en evidencia problemas en torno a vivienda, empleo, salud, educación y necesidades básicas que representan un riesgo para el logro del bienestar biopsicosocial de 
ellas y sus familias. Esta ha sido una situación que tiene como resultado la conformación de las diferentes asociaciones de Mujeres Madre Cabeza de Familia quienes se han convencido de la necesidad de agruparse para la gestión y realización de actividades que contribuyan al progresivo mejoramiento de su calidad de vida.

Los resultados afirman lo planteado por Sarason como lo cita Jariego Isidro M (2005), quien plantea que el sentido de comunidad es una experiencia subjetiva de pertenencia a una colectividad mayor, formando parte de una red de relaciones de apoyo mutuo en la que se puede confiar. ${ }^{12}$

\section{Percepción de las mujeres madre cabeza de familia frente al involucramiento}

Las mujeres participantes en el estudio se caracterizaron por tener un sentido de Involucramiento marcado, siendo de gran significancia que el $73 \%$ asegura estar de acuerdo y fuertemente de acuerdo en que la población femenina pertenece a grupos comunitarios, de participación comunitaria, de ayuda o Comités de Participación Comunitaria, un $50 \%$ que discuten con representantes de instituciones sobre asuntos de salud y un $61 \%$ que toman decisiones en el barrio continuamente.

Como lo afirma Stephanie Riger, citado por León Magdalena (1997) "la confianza en las instituciones sociales, la utilización máxima de los apoyos interpersonales e institucionales, el compromiso con la denuncia de los hechos son estrategias más apropiadas para los individuos de las clases medias y altas, cuyos intereses son atendidos por aquellas instituciones, que les brindan apoyo social y que pueden multiplicar los recursos y contactos disponibles, puede llevarlos, de hecho, a lograr no sólo el cambio personal sino también un cambio de estructura" ${ }^{13}$.

\section{Percepción de las mujeres madre cabeza de familia frente a la participación}

Se encontró que las madres encuestas expresan que las mujeres de su comunidad participan en actividades que incrementan su bienestar, ocupando cargos de liderazgo como se observa en el cuadro 2 donde el $74 \%$ de las encuestadas afirman estar en acuerdo y fuertemente de acuerdo en que las mujeres del barrio ocupan este tipo de cargos en su comunidad. Sin embargo creen que es notoria la ausencia de las mismas en otros grupos organizados que trabajan en beneficio de su sector, evidenciado por un número no mayor al 50\% de mujeres que están de acuerdo o fuertemente de acuerdo que las mujeres del barrio hacen parte de estos grupos, limitando sus acciones solo al bienestar de las mujeres y sus familias.

Lo siguiente indica que las mujeres como actores sociales de la comunidad realmente toman parte en las deliberaciones y decisiones sobre cualquier problema que afecta a la comunidad incluyendo las decisiones sobre necesidades y prioridades, la auto formulación de planes y adopción de medidas que se ajustan a su entorno porque surgen del consenso de sus miembros que les ha permitido adquirir un sentido de responsabilidad con respecto a su propio bienestar y el del grupo, contribuyendo

\footnotetext{
${ }^{12}$ Op.cit JARIEGO, Isidro Maya. Sentido de comunidad y potenciación comunitaria [en línea]. 2005 Julio [citado en 16 jul. 2007 ]. Disponible en internet: URL:http://dialnet. unirioja.es/ ervlet/articulo? código=1197378. Pág. 189

${ }^{13}$ Citado LEÓN, Magdalena. Poder y empoderamiento de las mujeres, Bogotá. En revista Región y Sociedad. Volumen XI. Numero 18.ISSN 0188- 7408 .Sonora-España.1997.
} 
conscientemente y constructivamente en el proceso de desarrollode sucomunidad, muestra de ello es la conformación de grupos de trabajo en torno a necesidades como pavimentación de las calles y ayuda a enfermos.

Cuadro 2. Distribución de la percepción de las mujeres madre cabeza de familia frente a la participación. Cúcuta 2007.

\begin{tabular}{|l|c|c|c|c|c|c|c|c|c|c|}
\hline \multirow{2}{*}{ ITEMS } & \multicolumn{2}{|c|}{ FD } & \multicolumn{2}{|c|}{ D } & \multicolumn{2}{|c|}{ I } & \multicolumn{2}{|c|}{ DA } & \multicolumn{2}{c|}{ FDA } \\
\cline { 2 - 11 } & $\mathrm{F}$ & $\%$ & $\mathrm{~F}$ & $\%$ & $\mathrm{~F}$ & $\%$ & $\mathrm{~F}$ & $\%$ & $\mathrm{~F}$ & $\%$ \\
\hline $\begin{array}{l}\text { Ocupación de } \\
\text { cargos de liderazgo }\end{array}$ & 13 & $4 \%$ & 30 & $10 \%$ & 39 & $12 \%$ & 161 & $51 \%$ & 72 & $23 \%$ \\
\hline $\begin{array}{l}\text { Ocupan cargos } \\
\text { en comités de salud }\end{array}$ & 27 & $8 \%$ & 46 & $15 \%$ & 105 & $33 \%$ & 103 & $33 \%$ & 34 & $11 \%$ \\
\hline $\begin{array}{l}\text { Juntas o comités } \\
\text { civicos }\end{array}$ & 10 & $3 \%$ & 16 & $5 \%$ & 143 & $45 \%$ & 110 & $35 \%$ & 36 & $12 \%$ \\
\hline $\begin{array}{l}\text { Comité de } \\
\text { participación } \\
\text { comunitaria }\end{array}$ & 13 & $4 \%$ & 16 & $5 \%$ & 145 & $46 \%$ & 105 & $33 \%$ & 36 & $12 \%$ \\
\hline $\begin{array}{l}\text { Junta } \\
\text { Administradora } \\
\text { Local (JAL) }\end{array}$ & 15 & $5 \%$ & 24 & $7 \%$ & 172 & $55 \%$ & 72 & $23 \%$ & 32 & $10 \%$ \\
\hline $\begin{array}{l}\text { Presentan solicitudes } \\
\text { escritas con relación } \\
\text { a asuntos como } \\
\text { educación u otro } \\
\text { asunto. }\end{array}$ & 16 & $5 \%$ & 30 & $10 \%$ & 95 & $30 \%$ & 123 & $39 \%$ & 51 & $16 \%$ \\
\hline $\begin{array}{l}\text { Organizan grupos } \\
\text { de trabajo en } \\
\text { torno a algún } \\
\text { asunto o necesidad. }\end{array}$ & 18 & $5 \%$ & 15 & $5 \%$ & 48 & $15 \%$ & 150 & $48 \%$ & 84 & $27 \%$ \\
\hline
\end{tabular}

FD: Fuertemente en desacuerdo D: En desacuerdo I: Indecisa DA: De acuerdo FDA: Fuertemente de acuerdo F: Frecuencia \%: Porcentaje.

En promoción de la salud, la participación comunitaria es un proceso por el cual los individuos y las familias toman a cargo su propia salud y su bienestar, lo mismo que el de la comunidad de la cual ellos forman parte. ${ }^{14}$ Gracias a esta participación, los individuos y los grupos sociales pueden incrementar sus responsabilidades en salud contribuyendo a un desarrollo más global, durante el desarrollo de la investigación se observo en las madres cabeza de familia la capacidad para empoderarse de su salud y la de sus familias, mediante la adopción de conductas que promocionan la misma y a realizar acciones para exigir sus derechos en las instituciones de salud.

Kate Young, citado en León Magdalena (1997) ${ }^{15}$, afirma que el proceso de mujeres que trabajan juntas y resuelven problemas basándose en el ensayo y el error, aprendiendo a través de la práctica, aprendiendo también a identificar aliados y a forjar alianzas cuando son necesarias, conducirá al empoderamiento tanto a nivel colectivo como individual. Es este proceso de participación, el que ha logrado que las madres cabeza de familia tomen parte en las decisiones y en los cambios, adquiriendo la capacidad de identificar necesidades comunes, que puedan ser atendidas, usando los recursos que le brinda su entorno.

\section{Percepción de las mujeres madre cabeza de familia frente a la influencia}

En el cuadro 3, se presenta la distribución de la percepción de las mujeres madres cabeza de familia sobre la influencia que estas tienen en la comunidad. Su participación activa en asuntos que afectan su salud, en política y en general en la búsqueda de bienestar para ellas y su entorno, se evidencian en este cuadro.

El empuje, la recursividad y creatividad son características que las mujeres expresan como las responsables de que un 75\% de ellas expresen estar de acuerdo y fuertemente de acuerdo en que las mujeres de su barrio pueden controlar o manejar adecuadamente las situaciones que a ellas les ocurren, evidenciado también en las diferentes formas de expresar su opinión con relación a lo que hacen los servicios de salud, ya que un $70 \%$ percibe estar de acuerdo

${ }^{14}$ CENTRO DE COOPERACIÓN INTERNACIONAL EN SALUD Y DESARROLLO. Avances [en línea]. 2004 Julio [citado en 18 jul. 2007]. Disponible en internet: URL:http://www.ccisd.org/esp/index2d.html

${ }^{15}$ Citado de LEÓN, Magdalena. Poder y empoderamiento de las mujeres. Ocipt. Pág. 110 


\section{Respuestas}

Año 13

No. 1

Junio 2008

ISSN 0122-820X

y fuertemente de acuerdo en que las mujeres recurren a diversos mecanismos que han logrado cambios positivos en los servicios que prestan las instituciones de salud en la comunidad.

Las mujeres cabeza de familia están vinculadas a un modelo social donde la participación en los asuntos políticos se convierte en un requisito para el disfrute de los recursos a los que han hecho merito a través de su liderazgo, se sustenta esto con los resultados obtenidos en los que el 68\% de las mujeres afirman tener intereses en asuntos políticos y el $75 \%$ afirman tener conocimiento y habilidad en asuntos políticos.
Esto confirma que cada vez se reconoce más el hecho de que las mujeres deberán conformar sus propias organizaciones con el fin de hacer que sus preocupaciones hagan parte de las prioridades en la agenda política. Como lo afirma Kate Young citado por León Magdalena (1999), la voluntad política florece cuando existe ímpetu político, sin embargo para adquirir suficiente poder político las organizaciones de mujeres deberán tratar de ampliar sus contactos con otras organizaciones que trabajan con miras a la transformación social. Es en este sentido que el empoderamiento de las mujeres resulta altamente relevante como herramienta imprescindible para su propio desarrollo. ${ }^{16}$

Cuadro 3. Distribución de la percepción de las mujeres madre cabeza de familia frente a la influencia.

Cúcuta 2007

\begin{tabular}{|c|c|c|c|c|c|c|c|c|c|c|}
\hline \multirow{2}{*}{ ITEMS } & \multicolumn{2}{|c|}{ FD } & \multicolumn{2}{|c|}{ D } & \multicolumn{2}{|c|}{ I } & \multicolumn{2}{|c|}{ DA } & \multicolumn{2}{|c|}{ FDA } \\
\hline & $\mathrm{F}$ & $\%$ & $\mathrm{~F}$ & $\%$ & $\mathrm{~F}$ & $\%$ & $\mathrm{~F}$ & $\%$ & $\mathrm{~F}$ & $\%$ \\
\hline $\begin{array}{l}\text { Tienen influencia en las decisiones que } \\
\text { afectan su salud }\end{array}$ & 15 & $5 \%$ & 28 & $9 \%$ & 87 & $28 \%$ & 124 & $39 \%$ & 61 & $19 \%$ \\
\hline $\begin{array}{l}\text { Han obtenido cambios notorios } \\
\text { en su barrio ocomunidad }\end{array}$ & 13 & $4 \%$ & 28 & $9 \%$ & 61 & $19 \%$ & 153 & $49 \%$ & 60 & $19 \%$ \\
\hline $\begin{array}{l}\text { Planean y realizan actividades con el fin } \\
\text { de ejercer influencia en las decisiones } \\
\text { importantes que se toman con relación } \\
\text { a asuntos civicos }\end{array}$ & 14 & $4 \%$ & 23 & $7 \%$ & 65 & $21 \%$ & 143 & $46 \%$ & 70 & $22 \%$ \\
\hline $\begin{array}{l}\text { Se sienten satisfechas por la influencia } \\
\text { que ellas tienen en la toma de decisiones } \\
\text { en los asuntos que afectan su salud }\end{array}$ & 16 & $5 \%$ & 34 & $11 \%$ & 83 & $26 \%$ & 124 & $39 \%$ & 58 & $19 \%$ \\
\hline $\begin{array}{l}\text { Diferentes formas de expresar su opinión } \\
\text { con relación a lo que hacen los servicios } \\
\text { de salud. }\end{array}$ & 14 & $4 \%$ & 30 & $10 \%$ & 51 & $16 \%$ & 146 & $46 \%$ & 74 & $24 \%$ \\
\hline $\begin{array}{l}\text { Tienen interés } \\
\text { en asuntospolíticos }\end{array}$ & 22 & $7 \%$ & 10 & $3 \%$ & 71 & $22 \%$ & 122 & $39 \%$ & 90 & $29 \%$ \\
\hline $\begin{array}{l}\text { Tienen conocimiento y habilidad } \\
\text { para trabajar en asuntos políticos. }\end{array}$ & 17 & $6 \%$ & 14 & $4 \%$ & 47 & $15 \%$ & 142 & $45 \%$ & 95 & $30 \%$ \\
\hline $\begin{array}{l}\text { Tienen influencia en la toma de } \\
\text { decisiones en asuntos de interés } \\
\text { para ellas }\end{array}$ & 14 & $4 \%$ & 7 & $2 \%$ & 52 & $17 \%$ & 155 & $49 \%$ & 87 & $28 \%$ \\
\hline $\begin{array}{l}\text { Pueden controlar o manejar } \\
\text { adecuadamente las situaciones } \\
\text { que a ellas les ocurren }\end{array}$ & 21 & $7 \%$ & 8 & $2 \%$ & 49 & $16 \%$ & 155 & $49 \%$ & 82 & $26 \%$ \\
\hline $\begin{array}{l}\text { Es mucho lo que las mujeres } \\
\text { pueden hacer para cambiar } \\
\text { las cosas que les ocurren a ella }\end{array}$ & 20 & $6 \%$ & 5 & $2 \%$ & 30 & $10 \%$ & 140 & $44 \%$ & 120 & $38 \%$ \\
\hline
\end{tabular}

FD: Fuertemente en desacuerdo D: En desacuerdo I: Indecisa DA: De acuerdo FDA: Fuertemente de acuerdo F: Frecuencia $\%$ : Porcentaje.

${ }^{16}$ Citado por LEÓN, Magdalena. Poder y empoderamiento de las mujeres. Ibíd. Pág. 114 


\section{Percepción de empoderamiento comunitario de las mujeres madre cabeza de familia}

En las mujeres madres cabeza de familia se encontró una alta percepción de empoderamiento comunitario (Véase cuadro 4), ya que de cada 100 mujeres encuestadas 63 presentaron puntajes por encima del $70 \%$ del total, es decir valores por encima de 95 . La media de la percepción del empoderamiento para el total de la muestra fue de 105 con una desviación estándar de 11. El 50\% de las mujeres presentaron puntajes menores o iguales a 105 equivalente a la mediana con una desviación estándar de 2 que indica una alta percepción de empoderamiento. El valor puntual que se presentó con mayor frecuencia en las encuestadas es de 101 con una desviación de 1 .

Cuadro 4. Distribución de la percepción de empoderamiento comunitario en las madres cabeza de familia. Cúcuta 2007

\begin{tabular}{|l|c|c|}
\hline $\begin{array}{l}\text { PERCEPCIÓN } \\
\text { DE EMPODERAMIENTO } \\
\text { COMUNITARIO }\end{array}$ & Frecuencia & Porcentaje \\
\hline ALTO & 200 & $63,50 \%$ \\
\hline MEDIO & 114 & $36,19 \%$ \\
\hline BAJO & 1 & $0,31 \%$ \\
\hline Total de mujeres & 315 & $100 \%$ \\
\hline
\end{tabular}

En el cuadro 4 , se puede observar que el $75 \%$ presentó una percepción de empoderamiento comunitario menor del $80 \%$ y mayor que el $74 \%$ lo que equivale a una percepción de empoderamiento alto. Al comparar estos resultados con los obtenidos en el estudio realizado por Gladys Canaval (1996), es notoria la diferencia, ya que en las mujeres de la muestra se aprecia una percepción baja del empoderamiento comunitario.

Como lo afirma Batliwala citado por León Magdalena(1997) “...el proceso de empoderamiento tiene que permitir a las mujeres organizarse en colectividades para que, de esta manera, rompan con el aislamiento individual y creen un frente unido por medio del cual puedan desafiar su subordinación. Así podrán iniciar la acción.”"17

Es claro que las Mujeres Madre Cabeza de Familia han tomado control sobre los recursos a los cuales los entes locales les ha permitido tener acceso, y se han apoderado de ellos obteniendo un sin número de beneficios, entre ellos el empoderarse ganando comprensión y control sobre las fuerzas personales, sociales, económicas y políticas reflejado en el progresivo mejoramiento de su situación, convirtiéndose de la misma manera en modelo para los sectores no organizados de mujeres que les den una mayor claridad de su poder transformador para cambiar los modelos sociales y crear condiciones propicias para su desarrollo dentro de la sociedad que tanto las ha marginado.

Esta situación encontrada en las mujeres, en términos de eficacia, significa que "el ver a otras mujeres con éxito, aumenta la creencia en el éxito personal; por el contrario, observar que otras fallan a pesar de grandes esfuerzos, disminuye el juicio sobre la propia eficacia y el nivel de motivación", y es ésta la razón vital que mantiene el espíritu combatiente y transformador en las mujeres madre cabeza de familia, que se ven reflejadas en el éxito de otras personas de su mismo sexo que han desarrollado papeles como líderes, logrando

${ }^{17}$ Citado por LEÓN, Magdalena. Poder y empoderamiento de las mujeres. Ibíd. Pág. 201 
múltiples beneficios que han contribuido al desarrollo personal y colectivo en los sectores bajo en los cuales ejerce su influencia. ${ }^{18}$

La alta percepción de empoderamiento hallada en las mujeres del estudio muestra inicios con relación a las mujeres de Cúcuta, para lograr a futuro dejar de ser una sociedad cerrada, la cual se define según Freire citado por León Magdalena(1997) como sociedades que producen una conciencia carente de una percepción estructural, el resultado es una "cultura de silencio" en la cual los dominados siguen siendo pasivos y permanecen sumergidos en una percepción fatalística de su situación ${ }^{19}$.

La ciudad junto con sus integrantes está logrando su apertura conllevando a la generación de cambios de conciencia y la adquisición de la capacidad de avanzar hacia una autonomía creativa que le está permitiendo a Cúcuta y a sus mujeres surgir de una civilización rodeada de conflictos, violencia e indiferencia que se ha mantenido a través de la historia y que hoy con el esfuerzo de grandes mujeres han posibilitado iniciar transformaciones concretas, como por ejemplo, la participación de las mujeres en actividades que incrementan el bienestar de la comunidad al liderar asociaciones de mujeres en cada localidad, que de igual forma están mejorando sus oportunidades en los distintos ámbitos de la sociedad.

42 su responsabilidad individual, social y de su capacidad para intervenir y orientar los cambios necesarios en el diseño y ejecución de las políticas en salud a través de la formulación eficaz de sus necesidades y de sus propuestas de mejora hacia este sector". ${ }^{20}$

Con la falta de creencia de algunas mujeres en sus capacidades para afectar el cambio a lo largo de la historia, ha hecho que mujeres como lo son las madres cabeza de familia vean el empoderamiento como una oportunidad útil que les abre las puertas hacia la concientización y el compromiso para el mejoramiento de su calidad de vida alterando su involucramiento en esfuerzos colectivos más activos que han logrado modificar los modelos tradicionales de la sociedad.

En Cúcuta la motivación de participación de la mujer en asociaciones ha sido promovida por la secretaria de equidad y genero, quien ha reconocido la importancia de facilitar espacios y condiciones que permitan a las mujeres modifiquen la imagen de sí mismas, las creencias sobre sus derechos y capacidades y desafien los sentimientos de inferioridad como lo cita León Magdalena (1997) ${ }^{21}$

El empoderamiento asumido por la colectividad femenina de la ciudad de Cúcuta es la clara declaración de la lucha contra el individualismo que como lo define Schuler citado por León Magdalena(1997), "supone sujetos independientes, autónomos, seguros de sí mismos y con un sentido de dominio y separación que deja a un lado aspectos de influencia social"; las mujeres madre cabeza de familia han asimilado el empoderamiento

Para el logro de esta igualdad, no solo es necesario que se modifique la percepción de la sociedad hacia la mujer, sino que la mujer "tome conciencia de su situación, de

\footnotetext{
${ }^{18}$ CANAVAL, Gladys Eugenia. Promoción de la salud: predictores de empoderamiento comunitario en mujeres. Op.cit. Pág. 5

${ }^{19}$ Citado por LEÓN, Magdalena. Poder y empoderamiento de las mujeres. Ocipt. Pág. 35, 39

${ }^{20} \mathrm{CEPAL}$. Políticas para el empoderamiento de las mujeres como estrategia de la lucha contra la pobreza [en línea]. Puerto España (Trinidad y Tobago), 11 de oct. 2001 [citado en 16 feb. 2006]. Disponible de Internet: <URL:http://www.eclac.org/mujer/noticias/noticias/7/9337/papermontanopobreza.pdf

${ }^{21}$ Citado por LEÓN, Magdalena. Poder y empoderamiento de las mujeres. Ibíd. Pág. 12
} 
como un proceso integrado con la comunidad, la cooperación y la solidaridad, haciendo de este grupo organizado un fuerte colectivo que ha demostrado su poder transformador en el mejoramiento de las condiciones a las que la sociedad desde tiempo atrás las ha venido sometiendo. ${ }^{22}$

En esta visión de empoderamiento colectivo las mujeres se tornan empoderadas a través de la toma de decisiones colectivas y dentro de los parámetros que juegan un papel primordial en la cohesión de grupo y la promoción de soluciones que beneficien a cada una de sus integrantes, este trabajo conjunto ha evidenciado el gran aporte que se puede obtener con la incorporación de los talentos de las mujeres a la gestión y a la dirección de proyectos que beneficien a la sociedad que se fortalece con el constante apoyo de instituciones gubernamentales que les ofrecen las herramientas necesarias para perpetuar este proceso de empoderamiento que aún comienza a fortalecerse en la sociedad cucuteña.

\section{Conclusiones}

El empoderamiento es una variable que se ve afectada por diversos factores que tiene que ver con los procesos de participación social, organización comunitaria y formación de coaliciones, es evidente en este estudio la influencia que tiene el pertenecer a grupos organizados, como lo son en este caso las asociaciones de madres cabeza de familia adscritas a la secretaría de equidad y género, esta se convierte en una condición influyente en el empoderamiento de las mismas, reflejado en los resultados que muestran una alta percepción de empoderamiento comunitario donde de cada 100 mujeres encuestadas 63 presentaron puntajes por encima del $70 \%$

Las mujeres participantes en el estudio en cuanto a la percepción de sentido de comunidad reconocieron que existen intereses comunes y la importancia de compartirlos para la organización de alianzas dirigidas a satisfacer esos intereses que les brinden mejores condiciones de vida y suplan su sed de superación.

En cuanto al involucramiento las madres cabeza de familia, refieren que las mujeres toman propiedad en las de decisiones que afectan su comunidad a través de su vinculación a grupos organizados donde se discuten y manifiestan inquietudes sentidas en la comunidad, además se conforman grupos de trabajo compartido con otros entes relacionados con estos intereses en el ámbito de la salud, educación, empleo y vivienda.

En relación a la participación, las mujeres como actores sociales de la comunidad realmente toman parte en las deliberaciones y decisiones sobre cualquier problema que afecta a la comunidad, incluyendo las decisiones sobre necesidades y prioridades, la auto-formulación de planes y adopción de medidas que se ajustan a su entorno.

Con respecto a la influencia, se debe destacar que las mujeres participantes en el estudio perciben un alto sentido de influencia en las mujeres de su barrio o comunidad lo que ha contribuido a la concepción, planeación, ejecución y aprovechamiento de planes y proyectos destinados a elevar sus propios niveles de vida, proporcionándoles oportunidades para implicarse en la solución de problemáticas compartidas por otras

${ }^{22}$ citado por de LEÓN, Magdalena. Poder y empoderamiento de las mujeres. Ibíd. Pág.22 
mujeres en su comunidad y aprender nuevas habilidades contribuyendo así a su proceso de empoderamiento comunitario y al incremento de la credibilidad en sus potenciales.

Es notable que el pertenecer a las asociaciones de madres cabeza de hogar produce un incremento en la confianza de las mujeres de la comunidad, convenciéndose de su propia capacidad para contribuir a enfrentar y resolver sus problemas comunes de salud, mediante su participación activa y responsable en el proceso de cambio, afirmando aún más su confianza con la obtención de resultados tangibles y transformadores en su localidad.

\section{Bibliografía}

ACEVEDO, Juan Francisco. El poder y el empoderamiento: entre la ciencia y la ideología [en línea]. España, 14 sep. 2004 [citado en 15 jun. 2006]. Disponible en internet:<URL: http://www. pucp.edu.es/-sentcom/poder. htm >

ALARCÓN GUISAO, Luz Mery. Marco legal sobre la equidad de género y derechos de

las mujeres [en línea]. Bogotá, 2003 [citado en 03 feb. 2007]. Disponible en internet: <URL: http://www. madellin.gov.co/metromujer/ consulta/sitema3>

ALDANA, Aura. Conferencia centroamericanay delCaribe: ciudad de género para alcanzar el desarrollo: el empoderamiento femenino como acción ciudadana: ética de una participación política diferente [en línea]. Nicaragua, 30 ago. 2002 [citado en 25 jun. 2006]. Disponible en internet: <URL:http:// www. cholonautas.edu.pe/conferencia $>$

\section{BARRICK GOLD CORPORATION.}

Reporte de Responsabilidad 2005 [online]. Barrik. [citado 18 Julio 2007]. Disponible de Internet. <http://www.barrick.com/theme/ barrick/files/ehss/online/2005/sp/sresp_2.asp.
COLOMBIA. CONSEJERÍA PRESIDENCIAL PARA LA EQUIDAD DE LA MUJER. Ley 823 del 2003 [en línea]. Bogotá, 2003 [citado en 03 feb. 2007]. Disponible en internet: <URL:http://www. presidencia.gov. co/equidad/acuerdo.htm $>$

COLOMBIA. IDEAM. Participación comunitaria: hacia la gobernabilidad y empoderamiento [en línea]. Bogotá, 2006 [citado en 03 feb. 2007]. Disponible en internet: <URL:http://www. ideam.gov.co>

CONSEJO ESPAÑOL DE LA MUJER. Plan de acción positiva para las mujeres: enfoque de género en las políticas públicas [en línea]. Madrid, 2004 [citado en 8 jun. 2006]. Disponible en internet:URL:http://www. emakunde.es/pagme/images/2_area_c:PDF\#s earch $=\% 22$ Empoderamiento

ESTADOS UNIDOS. COMITÉNACIONAL DE PROMOCIÓN DE LA SALUD. Lineamientos de política de promoción de la salud [en línea]. Estados Unidos, 2005 [citado en 03 ago. 2006]. Disponible en internet: <URL:http://www.bvs.org/ ConferenceonHealth Promotion $>$

ORGANIZACIÓN MUNDIAL DE LA SALUD. Promoción de la salud [en línea]. Ginebra, 2004 [citado en 8 ago. 2006]. Disponible de internet: URL:http://www.bvs. org.ar/pdf/glosario_sp.pdf.> 58

PLAN DE ACCION POSITIVA PARA LAS MUJERES EN LA C.A.E. Enfoque de género en las políticas públicas [online]. México. [citado 8 Jun. 2006 ]. Disponible de Internet: <http:www.emakunde.es/pagme/ images $/ 2$ area_c:PDF\# search $=\% 22 \mathrm{Empode}$ ramiento\%2oy\%20participaci\%C3\%B3n\%.

VANEGAS, PAKI. El empoderamiento de las mujeres: un camino hacia la equidad en el desarrollo [en línea]. México, feb. 2006 [citado en 3 jun. 2006]. Disponible en internet:<URL:http://www.nodo50.org/ aspa/module.php?link $=$ modules/desurasur/ articulo.php\&ID $=609>$ 